\title{
An associative analysis of instrumental biconditional discrimination learning
}

\author{
RUTH M. COLWILL and BETH A. DELAMATER \\ Brown University, Providence, Rhode Island
}

\begin{abstract}
Three different techniques were employed to analyze the associative structures mediating performance on an instrumental biconditional discrimination. In all three experiments, rats were trained concurrently on two tasks in which different stimuli signaled which one of two responses would be followed by reward. In each task, one response was rewarded in one stimulus and the other response was rewarded in the other stimulus. Correct responses earned pellets in one task and sucrose in the other task. The transfer procedure was used in Experiment 1A to identify whether or not an association developed between a biconditional discriminative stimulus and its instrumental outcome. Evidence was obtained that a biconditional cue elevated preferentially a new response trained with the same outcome. Experiments $1 \mathrm{~B}$ and 3 examined the potential contribution of this stimulus-outcome association to biconditional performance by training the biconditional cues as signals ( $\mathrm{S}-\mathrm{s}$ ) for the nonreinforcement of a different response. There was no evidence that this operation interfered with the ability of a biconditional cue to control performance of its correct response. In Experiments 18 and 2 , the value of the instrumental outcome was reduced in an attempt to assess the contribution of stimulus-response associations to performance on the biconditional discrimination. The results of Experiments $1 \mathrm{~B}$ and 2 reveal that correct responses were depressed following devaluation of the outcome used to train them, suggesting that learning about the response-outcome relation occurs. The implications of these results for binary and hierarchical models of instrumental learning are discussed.
\end{abstract}

In recent years, considerable progress has been made in identifying the content of the associations that mediate performance on simple instrumental discriminations. Two findings suggest that learning about a rewarding outcome occurs in a situation in which a stimulus $(\mathrm{S}+)$ signals when a response will be followed by that outcome. First, there is evidence that instrumental performance is affected by postconditioning manipulations of the value of the outcome (Colwill \& Rescorla, 1990a; Rescorla \& Colwill, 1989). Second, it has been shown that discriminative stimuli trained with one responseoutcome relation will selectively promote performance of other responses trained with the same outcome (Colwill \& Rescorla, 1988). These results have encouraged

This research was supported by National Science Foundation Grant IBN 8915342. We thank Eric Wolfinger for his careful assistance with data collection. Reports based on various portions of this work were presented at the 63rd Annual Meeting of the Eastern Psychological Association in Boston, April 1992; at the Centennial Meeting of the American Psychological Association in Washington, D.C., August 1992; and at the 33rd Annual Meeting of the Psychonomic Society in St. Louis, November 1992. A thesis based on Experiments $1 \mathrm{~A}$ and $1 \mathrm{~B}$ was submitted by B.A.D. to the Department of Psychology, Brown University, in partial fulfillment of the bachelor's degree requirements for honors in psychology, 1992. A brief report of Experiment 2 appears in Colwill (1993a, 1994). Correspondence concerning this article should be addressed to Ruth M. Colwill, Department of Psychology, Brown University, Box 1853, Providence, RI 02912.

-Accepted by previous editor, Vincent M. LoLordo the view that, over the course of $\mathrm{S}+$ training, associations develop between the instrumental response and the outcome ( $\mathrm{R}-\mathrm{O}$ association) and between the discriminative stimulus and the outcome ( $\mathrm{S}-\mathrm{O}$ association).

Other work has focused on an analysis of the associations learned in a situation in which a stimulus $(\mathrm{S}-$ ) signals when a response will not be followed by a rewarding outcome (Bonardi, 1989; Colwill, 1991). Two observations have been made about the operation of an $\mathrm{S}-$. First, several authors have found that an S- preferentially suppresses the response used for its training (Bonardi, 1989; Richeson \& Colwill, 1994). Moreover, suppression of the original response appears independent of both the value of the consequences associated with the original response and the availability of the outcome predicted by the stimulus (Colwill, 1991, 1993b). This evidence suggests that an $S$ - specifically inhibits its nonreinforced response $(S \dashv R)$. Second, it has been reported that the transfer of $S-s$ to new instrumental responses is mediated in part by the identity of the instrumental outcome. Colwill (1991) has shown that an Swill suppress better another response trained with the same outcome relative to a response trained with a different outcome. This finding of outcome-dependent transfer implies that an S- develops an inhibitory association with the instrumental outcome $(\mathrm{S} \dashv \mathrm{O})$.

The goal of the present experiments was to explore further the adequacy of an analysis of instrumental learning in terms of these types of binary associations. Of particular interest was the question of how animals 
solve an instrumental biconditional discrimination in which two stimuli differentially signal which one of two concurrently available responses will be reinforced and which will not (Trapold, 1970). In this kind of task, one response (R1) is rewarded only during one stimulus (S1) and the other response (R2) is rewarded only during the other stimulus (S2). The present paper reports a series of experiments that examined how rats learn which response to make in each stimulus when correct responses are followed by the same outcome $(\mathrm{O})$.

There are two straightforward versions of a binary associative analysis of performance on this simple biconditional discrimination. One approach employs a mixture of $\mathrm{R}-\mathrm{O}, \mathrm{S}-\mathrm{O}$, and $\mathrm{S} \dashv \mathrm{R}$ associations. According to this view, performance of the correct response is attributed to a combination of $\mathrm{S}-\mathrm{O}$ and $\mathrm{R}-\mathrm{O}$ associations. Essentially, it is assumed that discriminative stimuli control the responses with which they are trained in the same way as they transfer to or promote performance of new instrumental responses trained with the same outcome. This assumption is supported by recent work showing that instrumental discriminative stimuli depend upon an intact $\mathrm{S}-\mathrm{O}$ association to control performance of their original responses (Colwill, 1993b). However, in order to generate differential responding in the presence of the biconditional discriminative stimuli trained with a single outcome, promotion of the incorrect response by the $\mathrm{S}-\mathrm{O}$ association must be counteracted. One way to accomplish this is through an inhibitory association between the stimulus and the incorrect response (Bonardi, 1989; Colwill, 1991).

The other version of a binary account of biconditional discrimination learning also makes use of an association between the discriminative stimulus and the response. However, correct performance is attributed to the development of excitatory S-R associations. Because rewards only follow performance of the correct response in each stimulus, an association between the discriminative stimulus and the correct response will be selectively strengthened (e.g., Hull, 1943). In this way, the unique S-R associations guarantee performance of the correct response in each stimulus. What is important to note about this classical S-R account is that the outcome, although responsible for producing the association that permits this problem to be solved, is not itself represented in that associative structure. Consequently, manipulations of the value of the outcome after learning has taken place will have no differential impact on biconditional discriminative performance.

The present experiments employed three different techniques to analyze the associative structures mediating performance on a biconditional discrimination task. First, the transfer procedure was used in Experiment $1 \mathrm{~A}$ to identify whether or not an association developed between the biconditional discriminative stimulus and the instrumental outcome. Second, in Experiments $1 \mathrm{~B}$ and 3 , the biconditional cues were retrained as signals $(S-s)$ for the nonreinforcement of a different response to as- sess the involvement of the potential $\mathrm{S}-\mathrm{O}$ association in performance of the correct response on the biconditional discrimination. This operation has been shown to produce a virtual elimination of the ability of a discriminative stimulus to control its original response when the outcome employed for $\mathrm{S}$ - training is the same as that used for original discrimination training (Colwill, 1993b). Third, Experiments $1 B$ and 2 manipulated the value of the instrumental outcome in an attempt to assess the contribution of excitatory $\mathrm{S}-\mathrm{R}$ associations to performance on the biconditional discrimination.

\section{EXPERIMENT 1A}

The purpose of Experiment 1A was to use the transfer procedure to assess the presence of $\mathrm{S}-\mathrm{O}$ associations in biconditional discrimination learning. Rats were trained concurrently on two independent biconditional discrimination tasks. In one task using auditory discriminative stimuli, one response (R1) was rewarded with one outcome (O1) in the presence of one stimulus (A1), and the other response (R2) was rewarded with the same outcome $(\mathrm{O} 1)$ in the presence of the other stimulus (A2). In the other task using visual discriminative stimuli, R1 was rewarded with a different outcome (O2) in the presence of one stimulus (V1), and R2 was rewarded with that outcome $(\mathrm{O} 2)$ in the presence of the other stimulus (V2).

Following acquisition of the biconditional discriminations, two new instrumental responses were trained, one (R3) with $\mathrm{O} 1$ and one (R4) with O2. Each discriminative stimulus was then tested with the two new responses. If information about outcome identity is provided by the biconditional discriminative stimuli, then each stimulus should selectively promote performance of the response that was trained with the same outcome. Thus, A1 and A2 should elevate R3, whereas V1 and V2 should elevate R4. The basic design of the biconditional training procedure and the transfer test is shown in Figure 1.

\section{Method}

\section{Subjects}

The subjects were 16 experimentally naive, male Holtzmanderived Sprague-Dawley rats (Harlan Co.) approximately 100 days old at the start of the experiment. They were housed individually. Water was always available in the home cage. Daily food intake was regulated so that the animals were maintained at $80 \%$ of their free-feeding body weight.

\footnotetext{
Apparatus

The apparatus consisted of eight identical Skinner boxes measuring $22.9 \times 20.3 \times 20.3 \mathrm{~cm}$. The two end walls of the chamber were aluminum, and the side walls and ceiling were made of Plexiglas. The floor of the chamber was composed of $0.48-\mathrm{cm}$ stainless steel rods spaced $1.9 \mathrm{~cm}$ apart. Each chamber had a recessed food magazine in the center of one end wall. Sucrose and pellets were delivered through separate tubes inserted through the roof of the food magazine. Each operation of the sucrose dispenser allowed $0.2 \mathrm{ml}$ of $8 \%$ sucrose to flow onto the floor of the food magazine,
} 


\section{Basic design of Experiment 1A \\ Discrimination Training and Transfer Test}

\author{
Discrimination \\ Training
}

A1: R1-O1, R2A2: R2-O1, R1-

V1: R1-O2, R2-

V2: R2-O2, R1-

\author{
Transfer \\ Training
}

$\mid$

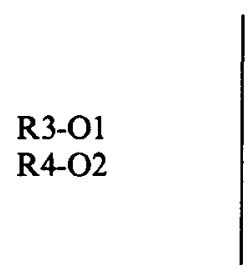

Transfer

Testing

A1: R3 $\vee$ R4
V2: R3 $v$ R4

V1: R3 v R4

A2: R3 v R4

Figure 1. Basic design of biconditional discrimination training and the transfer test in Experiment $1 \mathrm{~A}$. $\mathrm{A1}$ and $\mathrm{A} 2$ denote auditory discriminative cues (noise and tone), and V1 and V2 denote visual discriminative cues (steady light and flashing light). R1, R2, R3, and R4 are instrumental responses (nose poke, handle pull, lever press, and chain pull). $\mathrm{O} 1$ and $\mathrm{O} 2$ are reinforcers (food pellets and liquid sucrose).

where it collected in a shallow indentation. Each operation of the pellet dispenser allowed a single $45-\mathrm{mg}$ food pellet (Formula A, P. J. Noyes Co.) to drop onto the floor of the magazine.

Each box was equipped with four manipulanda: a lever, a chain pull, a nose poke, and a handle pull. The lever was mounted $2.5 \mathrm{~cm}$ from the right-hand wall of the food magazine. The chain was suspended from a microswitch mounted on the roof of the chamber. The end of the chain was $11 \mathrm{~cm}$ from the grid floor and $3 \mathrm{~cm}$ from the left-hand wall of the food magazine. Located $5.5 \mathrm{~cm}$ directly above the roof of the magazine was the nose-poke manipulandum, which consisted of a circular aperture, $2 \mathrm{~cm}$ in diameter and $1.3 \mathrm{~cm}$ deep. The back of this aperture was covered by a metal plate that operated a microswitch whenever it was depressed. Mounted on the same side of the chamber as the chain, but $1.5 \mathrm{~cm}$ below the grid floor, was the handle-pull manipulandum. This consisted of a short flat rod protruding $3 \mathrm{~cm}$ into the chamber. Whenever the rod was pulled upward, a microswitch was closed and a response recorded. The same model of microswitch (Unimax Switch Co., 2HBT-1) was used to detect responding on all four manipulanda. Access to these manipulanda was prevented by covering the lever with a metal plate or by retracting it, by retracting the chain through an opening in the ceiling, by inserting a metal cover into the aperture of the nose-poke manipulandum, and by withdrawing the arm of the handle pull.

Each Skinner box was enclosed in a sound-attenuating and light-resistant shell. Two loudspeakers were mounted on the back wall inside the shell, one in the upper left-hand corner and one in the upper right-hand corner. One speaker permitted presentation of a white noise $(\mathrm{N})$, and the other speaker allowed presentation of an $1800-\mathrm{Hz}$ tone (T). A $6-\mathrm{W}$ houselight $(\mathrm{F})$ was mounted from the ceiling of each shell such that it was located over the center of the operant chamber. It was flashed once per second. Another 6-W light (L) was mounted on the side wall of each operant chamber about $3 \mathrm{~cm}$ above the grid floor. Experimental events were controlled and recorded automatically by interfacing (Med Associates) and an XT microprocessor located in an adjoining room.

\section{Procedure}

Magazine training. The subjects were given one session of magazine training in which 10 food-pellet reinforcers followed by 10 liquid-sucrose reinforcers were delivered on a variable-time (VT) 60-sec schedule. No manipulanda were available during this session.

Response training. All subjects were then trained to nose poke and handle pull for pellet and sucrose rewards. Initially, each re- sponse was trained on a continuous reinforcement (CRF) schedule until 30 reinforcers had been earned. The subjects were trained to nose poke for pellets in the first session, to handle pull for pellets in the second session, to nose poke for sucrose in the third session, and to handle pull for sucrose in the fourth session.

Following CRF training, the subjects received two 20-min sessions with each response-outcome combination. In each session, responding was reinforced on a variable-interval (VI) $30-\mathrm{sec}$ schedule. The first four sessions occurred in the following sequence: handle pulling for sucrose, nose poking for pellets, handle pulling for pellets, and nose poking for sucrose. The next four sessions were given in the reverse order.

Biconditional discrimination training. The subjects were trained concurrently on two biconditional discrimination tasks. For one task, two visual stimuli ( $\mathrm{L}$ and $\mathrm{F}$ ) served as the conditional cues. For the other task, two auditory stimuli ( $\mathrm{T}$ and $\mathrm{N}$ ) served as conditional cues. Training sessions were administered daily, and the order of training on the two tasks followed a repeating ABBABAAB sequence. The subjects received a total of 14 sessions of training on each task.

For both tasks, each training session contained 1630 -sec presentations of each of the two stimuli. Both the nose-poke and the handle-pull responses were available in these sessions. A response was reinforced on a VI 30 -sec schedule only in the presence of its correct stimulus. Food pellets served as the reinforcer for one biconditional discrimination task, and sucrose liquid served as the reinforcer for the other biconditional discrimination task. Nose poking was designated as the correct response for one of the auditory and one of the visual stimuli; handle pulling was designated as the correct response for the other auditory and visual stimuli. The intertrial interval (ITI) was gradually lengthened over the course of training. For the first training session with each pair of discriminative stimuli, the mean ITI was $15 \mathrm{sec}$. For the next four sessions on each task, it was lengthened to a mean of $30 \mathrm{sec}$; for the next seven sessions on each task, the mean ITI was $60 \mathrm{sec}$; and for the final two sessions on each task, it was increased to a mean of $90 \mathrm{sec}$.

The specific designations of correct responses and outcome identities were counterbalanced across animals in the following way. Half of the animals always earned pellets during the sessions with the auditory cues and sucrose during the sessions with the visual cues; this contingency was reversed for the remaining animals. Within each of these conditions, half of the animals were rewarded for nose poking in the noise and in the steady light and for handle pulling in the tone and in the flashing light. The remaining 
animals were rewarded for handle pulling in the noise and in the steady light and for nose poking in the tone and in the flashing light.

Because of initial low levels of responding by some animals, remedial sessions of VI training were administered to elevate responding. These sessions were given after two sessions of conditional discrimination training on each task had been completed. An additional VI training session with the sucrose reward was also given after five further sessions on each conditional discrimination task.

Transfer-response training. Two new responses, lever pressing and chain pulling, were then trained. Each response was trained initially on a CRF schedule until 30 reinforcers had been earned. The subjects were then given one 20 -min session of training with each response in which its outcome was available on a VI 30 -sec schedule. This was followed by four 20 -min sessions in which both responses were available, but each was reinforced on separate VI $60-\mathrm{sec}$ schedules. For half of the animals, pellets served as the reinforcer for lever pressing and sucrose served as the reinforcer for chain pulling. The reverse was true for the remaining animals. Following this training, the subjects were given an 8-min extinction session in which both responses were available.

Transfer testing. The discriminative stimuli were examined for their ability to transfer to the lever and chain in a series of test sessions. In each test session, both the lever and the chain were available but responding was never reinforced. Within each session, there were an equal number of presentations of one visual and one auditory stimulus with a mean $30-\mathrm{sec}$ ITI; in this way, each session contained one stimulus trained with pellets and one stimulus trained with sucrose.

In the first test session with each pair of auditory and visual stimuli, there were four $30-\mathrm{sec}$ presentations of each stimulus scheduled in an $\mathrm{ABBABAAB}$ sequence. In the second test session with each pair of auditory and visual stimuli, there were eight 30-sec presentations of each stimulus. The first eight trials were scheduled in an ABBABAAB sequence; this sequence was reversed for the last eight trials. For all subjects, $\mathrm{N}$ and $\mathrm{F}$ were tested in the same session and $\mathrm{T}$ and $\mathrm{L}$ were tested together.

Between the first and second series of test sessions, the subjects received three sessions of biconditional discrimination training with each of the two pairs of stimuli. In addition, each of the second test sessions was preceded by a 20 -min VI training session with the two transfer responses. The procedural details of these training sessions were identical to those described for original training. The purpose of these additional training sessions was to attenuate any decremental effects that might have been produced by initial testing.

\section{Results and Discussion}

\section{Biconditional Discrimination Training}

Acquisition of the biconditional discriminations proceeded smoothly; by the end of training, responding occurred predominantly during the stimulus when it was followed by a reward. Analysis of the terminal session of discrimination training collapsed across stimulus, response, and outcome identity revealed that performance of the correct response ( 25.0 responses per minute) was significantly higher than performance of the incorrect response (7.9 responses per minute) during the biconditional stimuli [Wilcoxon $T(16)=0, p<.01$ ]. Furthermore, relative to the ITI rate ( 3.2 responses per minute), the biconditional cues elevated performance of both the correct response $[T(16)=0, p<.01]$ and the incorrect response $[T(16)=0, p<.01]$. Similar results were also obtained from an analysis of the session of discrimina- tion training preceding the second transfer test. The rate of correct responses (22.8 responses per minute) was significantly higher than the rate of incorrect responses (7.5 responses per minute) and the ITI rate (2.0 responses per minute) $[T \mathrm{~s}(16)=0, p<.01]$. There were also more incorrect responses than ITI responses $[T(16)=0, p<.01]$.

\section{Transfer-Response Training}

Training on the transfer responses proceeded smoothly. On the last day of VI training, the mean rate of responding for pellets ( 10.7 responses per minute) was significantly higher than the mean rate of responding for sucrose ( 7.4 responses per minute) $[T(16)=22, p<.05]$. There was no significant difference between the rates of lever pressing and chain pulling (10.4 and 7.8 responses per minute, respectively). A similar pattern of results emerged during the 8-min extinction test. Responding trained with pellets was significantly higher than that trained with sucrose ( 11.5 and 8.2 responses per minute, respectively) $[T(15)=20, p<.05]$, and the rate of lever pressing (11.1 responses per minute) did not differ significantly from that of chain pulling ( 8.6 responses per minute). In the sessions of VI training that immediately preceded the second series of transfer testing, there was no significant effect of either response or outcome identity. The mean rates of lever pressing and chain pulling were 10.0 and 7.6 responses per minute, respectively; the mean rates of pellet and sucrose trained responses were 9.8 and 7.8 responses per minute, respectively.

\section{Transfer Testing}

The results of the first test session with each biconditional discriminative stimulus were moderately encouraging. Relative to the ITI rate $(4.5$ responses per minute), the discriminative stimuli promoted performance of the transfer response trained with the same outcome (5.7 responses per minute) $[T(16)=25, p<.05]$ but had no significant effect on the different response (5.0 responses per minute). However, comparison of the rates of same and different responses failed to reveal a significant difference.

Of primary interest are the results of the second transfer-test session shown in Figure 2. Responding is shown separately during the stimuli trained with the same outcome as the response (SAME), during stimuli trained with a different outcome (DIFF), and during the ITI. There was a substantial elevation of the same response relative both to the different response $[T(16)=$ $0, p<.01]$ and to the ITI rate $[T(16)=0, p<.01]$. Responding on the different response, however, did not differ significantly from the ITI $[T(16)=47, p>.10]$.

The pattern of these results is identical to that reported by Colwill and Rescorla (1988). The selective elevation of a new instrumental response as a function of shared association with an outcome suggests that the biconditional discriminative stimuli developed associations with the instrumental outcome. The present results, 


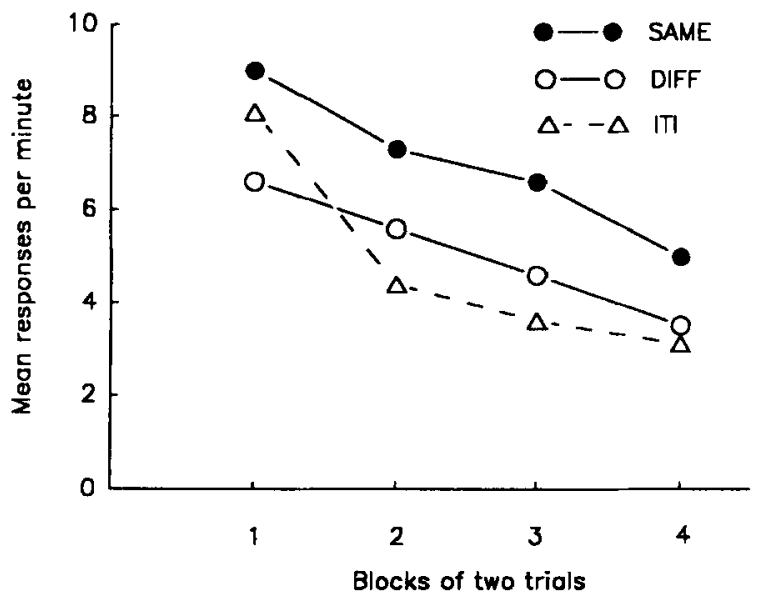

Figure 2. Transfer test results from Experiment 1A. Responding is shown when a stimulus signaled the same outcome as the transfer response (filled circles), a different outcome from the transfer response (open circles), and during the ITI (open triangles) when no stimulus was present.

together with those of Colwill and Rescorla (1988), encourage the view that subjects encode information about the identity of the outcome during training on a variety of appetitive instrumental tasks.

\section{EXPERIMENT 1B}

The first goal of Experiment $1 \mathrm{~B}$ was to evaluate the role of the $\mathrm{S}-\mathrm{O}$ association in performance on the biconditional discrimination. Recent evidence suggests that in simple instrumental discriminations performance of the response may be undermined by treatments that extinguish the $\mathrm{S}-\mathrm{O}$ association (Colwill, 1993b). In one study, Colwill (1993b) trained one discriminative stimulus with one response-outcome relation (S1: R1 $\rightarrow$ O1) and a different discriminative stimulus with another response-outcome relation ( $\mathrm{S} 2: \mathrm{R} 2 \rightarrow \mathrm{O} 2)$. Both stimuli were then trained as signals for the nonreinforcement of another instrumental response (R3) trained with one of these outcomes (either O1 or O2). Thus, one of the stimuli (same $\mathrm{S}+/ \mathrm{S}-$ ) signaled that $\mathrm{R} 3$ would not be followed by the outcome used for the original training of that stimulus, whereas the other stimulus (different $\mathrm{S}+/ \mathrm{S}-$ ) signaled the omission of a different responsecontingent outcome. In a subsequent extinction test with the original responses, the same $S+/ S-$ no longer evidenced an ability to elicit the response with which it had been trained, although the different $\mathrm{S}+/ \mathrm{S}-$ continued to evoke its original response. These results suggest that the $\mathrm{S}-\mathrm{O}$ association mediates performance of the instrumental response in a simple instrumental discrimination.

Experiment $1 \mathrm{~B}$ used this technique to modify the $\mathrm{S}-\mathrm{O}$ association in order to evaluate its contribution to performance on the biconditional discrimination task. The basic design of this stage of the experiment is shown in
Figure 3. Following retraining on the original discriminations, each of the four stimuli was trained as a signal $(\mathrm{S}-)$ for the nonreinforcement of one of the two transfer responses (R3 and R4). Two of the stimuli (one visual and one auditoky) were trained as signals for the omission of the same outcome used for their original training; the other two stimuli signaled the omission of a different outcome. Finally, the subjects were tested with the original responses ( $R 1$ and $R 2$ ) in the presence of each of the four stimuli. It was expected that if S-O associations contributed to performance of the original responses, the same $\mathrm{S}+/ \mathrm{S}-$ stimuli should be less effective than the different $S+/ S-$ stimuli in promoting their original responses.

The second goal of Experiment $\mathrm{IB}$ was to provide a preliminary assessment of the role of the outcome in determining performance on the biconditional discrimination. The outcome-revaluation procedure was used to probe the sensitivity of the correct response to the current value of its outcome. Because of the present subjects' extensive experience with the outcomes, a satiation procedure was used to decrease the attractiveness of the instrumental outcome. The subjects were tested on each biconditional discrimination first following satiation on one outcome and then following satiation on the other outcome. To the degree that S-R learning underlies instrumental performance on the biconditional task, there should be no differential effect on performance on the two tasks of devaluing the outcome for one of those tasks.

\section{Method}

\section{Subjects and Apparatus}

The subjects were the same as those used in Experiment $1 \mathrm{~A}$. They were housed and maintained according to the procedures described for Experiment 1A. The apparatus was also the same as that used in Experiment $1 \mathrm{~A}$

\section{Procedure}

Retraining of the biconditional discriminations. Using the procedure of Experiment 1A, two sessions of biconditional dis-

\author{
Basic design of Experiment 1B \\ S- Training and Testing
}

S- Training

R3-O1, A1: R3-, V2: R3-
R4-O2, V1: R4-, A2: R4-

Testing
A1: R1 v R2

V2: R1 V R2

V1: R1 v R2

A2: R1 v R2
Figure 3. Basic design of $S$ - training and the test of its effects on original biconditional discriminative performance in Experiment $1 \mathrm{~B}$. $A 1$ and $A 2$ denote auditory discriminative cues (noise and tone), and $\mathrm{V} 1$ and $\mathrm{V} 2$ denote visual discriminative cues (steady light and flashing light). R1, R2, R3, and R4 are instrumental responses (nose poke, handle pull, lever press, and chain pull). $O 1$ and $O 2$ are reinforcers (food pellets and liquid sucrose). 
crimination training with each pair of cues were given. The purpose of this retraining was to ensure reliable performance on the two discriminations after transfer testing.

Transfer-response retraining. The lever and chain responses were retrained separately for $10 \mathrm{~min}$. These responses were reinforced on a VI 30-sec schedule with the same outcomes used for their original training in Experiment $1 \mathrm{~A}$.

S- training. In this phase, the four biconditional discriminative stimuli were trained as signals for the omission of a response-contingent outcome that was either the same as or different from the outcome used in the original discriminations. Each session contained one auditory and one visual stimulus with one response manipulandum: in 16 sessions, $\mathrm{N}$ and $\mathrm{F}$ were trained with one response (either lever or chain), and in the other 16 sessions, $\mathrm{T}$ and $\mathrm{L}$ were trained with a different response (either chain or lever). Sessions were conducted daily and followed a repeating ABBABAAB sequence. Within each session, there were 1630 -sec presentations of each of the two stimuli separated by a mean ITI of $30 \mathrm{sec}$. The order of trial presentations was randomized in blocks of eight trials. Responses were reinforced on a VI 30-sec schedule, except during the stimulus presentations.

The various combinations of stimuli, responses, and outcomes were counterbalanced across subjects in the following way. For 4 of the animals, $\mathrm{N}$ and $\mathrm{F}$ signaled the omission of pellets for lever pressing, and $\mathrm{T}$ and $\mathrm{L}$ signaled the omission of sucrose for chain pulling; for four different animals, $\mathrm{N}$ and $\mathrm{F}$ signaled the omission of pellets for chain pulling, and $\mathrm{T}$ and $\mathrm{L}$ signaled the omission of sucrose for lever pressing; for another 4 animals, $\mathrm{N}$ and $\mathrm{F}$ signaled the omission of sucrose for lever pressing, and $\mathrm{T}$ and $\mathrm{L}$ signaled the omission of pellets for chain pulling; finally, for the remaining 4 animals, $N$ and $F$ signaled the omission of sucrose for chain pulling, and $\mathrm{T}$ and $\mathrm{L}$ signaled the omission of pellets for lever pressing.

Testing for control of original responses. Each of the four discriminative stimuli was tested with the original responses, nose poke and handle pull. The first test session contained four 30 -sec presentations of each of $\mathrm{N}$ and $\mathrm{F}$. The trial sequence was NFFNFNNF, and trials were separated by an ITI of $90 \mathrm{sec}$. The second test session conducted the following day was identical to the first, except that $T$ and $L$ were presented. The trial sequence was TLLTLTTL. During testing, responding was never reinforced.

Satiation and test. Two sessions of retraining were given on each biconditional task. The procedural details were the same as those described for original training.

The subjects were sated first on sucrose and then, on a different day, on pellets. Satiation was accomplished by delivering the designated outcome on a VT 30 -sec schedule until the animal had stopped consuming the reward. The subject was removed from the operant chamber but was replaced $5 \mathrm{~min}$ later and given additional deliveries of the to-be-sated outcome until consumption ceased. This cycle was repeated twice. During this satiation procedure, no manipulanda were available and no discriminative stimuli were presented.

The subjects were then removed from the chambers and placed in holding cages while the chambers were prepared for testing. The test session contained two presentations of each of the four discriminative stimuli separated by an ITI of $90 \mathrm{sec}$. The order of testing was NFFNTLLT following satiation on the sucrose, and TLLTNFFN following satiation on the pellets. Both the nose-poke and the handle-pull manipulanda were available in these test sessions, but responses were never reinforced.

\section{Results and Discussion}

\section{Retraining}

Retraining proceeded uneventfully. Performance on the last session of each discrimination was similar to that observed at the end of original training. Mean rates of correct and incorrect responses during a stimulus were 24.1 and 9.2 responses per minute, respectively. The mean rate was 2.6 responses per minute during the ITI. Analysis of these data revealed that the correct response was significantly elevated by its stimulus relative to performance of the incorrect response $[T(16)=0, p<$ $.01]$. Both correct and incorrect responses occurred at a higher rate during the stimuli than during the ITI $[T \mathrm{~s}(16)=0, p<.01]$.

Assignment of discriminative stimuli to $S$ - training with either the same or the different outcome matched performance of correct and incorrect responses in the final session of biconditional discrimination training. For the stimuli trained as $\mathbf{S}-\mathrm{s}$ with the same outcome, the mean rates of correct and incorrect responses were 25.7 and 9.3 responses per minute, respectively. For the stimuli trained as $\mathrm{S}-\mathrm{s}$ with a different outcome, the mean rates of correct and incorrect responses were 22.4 and 9.2 responses per minute, respectively. There were no significant differences in the rates of either correct or incorrect responses between the same and different conditions.

\section{S- Training}

The effects observed in the transfer tests persisted through the initial sessions of $S$ - training. In the first session of $\mathrm{S}$ - training, the mean rate of responding was higher during the stimulus that had been trained with the same outcome than during the stimulus trained with a different outcome (14.5 and 12.3 responses per minute, respectively) $[T(16)=7, p<.01]$. Relative to the ITI response rate (11.8), the stimulus trained with the same outcome increased responding $[T(16)=3, p<.01]$, but the stimulus trained with a different outcome had no significant effect.

Over the course of $\mathbf{S}$ - training, the level of responding during the ITI increased substantially, whereas responding during the stimuli declined slightly. Analysis of the terminal $\mathrm{S}$ - session revealed that responding during the ITI (19.2 responses per minute) was significantly higher than responding during either the same stimulus (11.5 responses per minute) or the different stimulus (9.9 responses per minute) $[T \mathrm{~s}(16)=1, p \mathrm{~s}<.01]$. Although the mean rate of responding during the same stimulus was numerically higher than that during the different stimulus, this difference was not significant $[T(16)=44, p>.10]$.

Suppression ratios for the first and last sessions of $S-$ training were calculated for each subject by dividing the rate of responding during the stimulus by the sum of that rate and the ITI rate. $S-$ training resulted in a significant increase in the suppression of responding during stimulus presentations relative to the ITI between the first and last training sessions (.53 and .35 , respectively) $[T(16)=0, p<.01]$.

\section{Testing for Control of Original Responses}

The results of the extinction test with the discriminative stimuli and their original responses are shown in Figure 4. Responding is shown in the presence of the two 
stimuli that were trained to signal the omission of an outcome that was either the same as (top panel) or different from (lower panel) the outcome used for original training. Within each panel, responding is shown separately for the correct response, the incorrect response, and during the ITI. Inspection of Figure 4 reveals that the discriminative stimuli continued to exhibit differential control over their original responses regardless of which outcome had been used for $\mathrm{S}-$ training. During presentations of stimuli trained as $S-s$ with the same outcome, performance of the correct response was significantly elevated relative to both the incorrect response $[T(16)=26, p<.05]$ and the ITI rate $[T(16)=$ $13, p<.01]$. Similar results were obtained when the stimuli had been trained as $\mathrm{S}-\mathrm{s}$ with a different outcome. Performance of the correct response was significantly elevated relative to both the incorrect response $[T(16)=20.5, p<.05]$ and responding during the ITI $[T(16)=24, p<.05]$. In neither condition did performance of the incorrect response differ significantly from the ITI $[$ Ts $(16)=45, p>.10]$. Furthermore, direct comparison of the rates of correct responding across the different- and same-outcome conditions yielded no significant difference $[T(16)=56, p>.05]$. In contrast to the results obtained by Colwill (1993b) with simple discriminative stimuli, there was no evidence that $\mathrm{S}$ - training with the same outcome had any adverse effect on the ability of biconditional stimuli to promote performance of their responses. Whereas Colwill (1993b) found that $\mathrm{S}$ - training with the same outcome removed the ability of that stimulus to increase performance of its response relative to the ITI, there was no evidence for a similar effect in the present experiment.

These results show that, regardless of the outcome used, the training of biconditional discriminative stimuli as signals for the nonreinforcement of an instrumental response does not affect their ability to promote their original reinforced responses. The failure to obtain an outcome-specific decremental effect of $S-$ training in this experiment suggests that performance of the correct response is not promoted by an $\mathrm{S}-\mathrm{O}$ association in this type of instrumental discrimination.

\section{Satiation and Test}

The retraining sessions were sufficient to reestablish good performance on the two tasks. Performance on the last session of each discrimination was similar to that observed at the end of original training. Mean response rates during a stimulus were 20.9 responses per minute for the correct response, 7.1 responses per minute for the incorrect response, and 2.7 responses per minute during the ITI. Analysis of these data revealed that the correct response was significantly elevated by its stimulus relative to performance of the incorrect response $[T(16)=$ $0, p<.01]$. Both correct and incorrect responses occurred at a higher rate during the stimuli than during the ITI $[T \mathrm{~s}(16)=1, p<.01]$.

The results of primary interest concern the effect of outcome satiation on performance of the correct re-
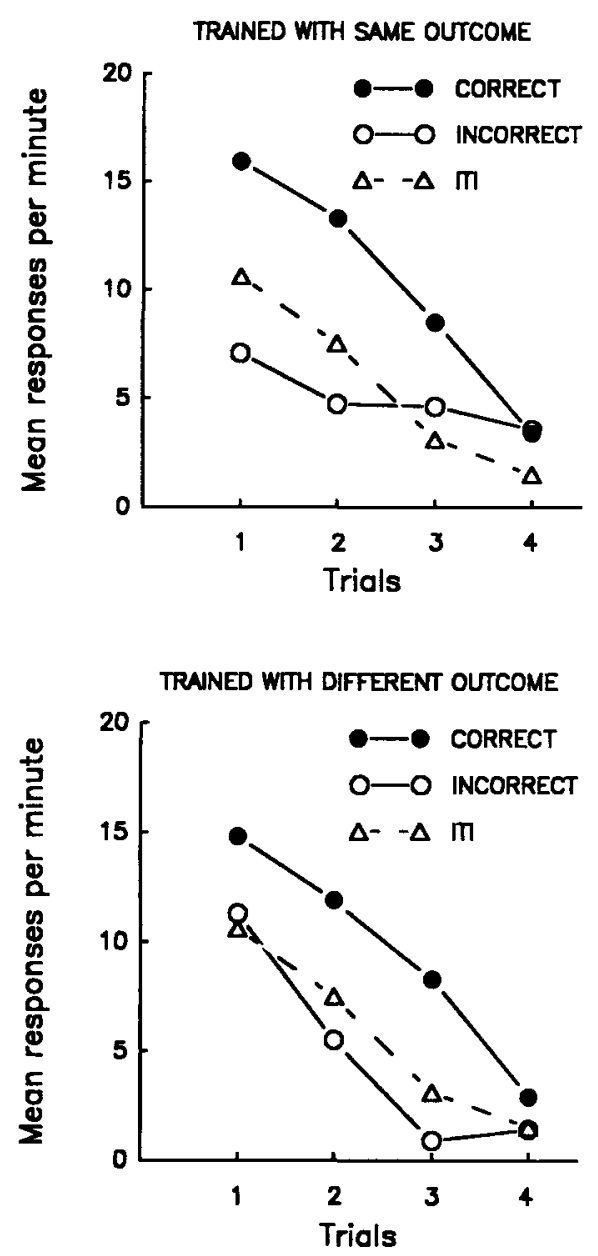

Figure 4. Mean rates of responding on the biconditional discriminations following $S$ - training in Experiment 1B. The top panel displays performance on the discrimination whose cues were trained with the same outcome used for $S-$ training; the bottom panel displays the comparable data when $S$ - training employed a different outcome. In each panel, correct (filled circles) and incorrect responses (open circles) are plotted separately. Responding is also shown during the ITI (open triangles) when no stimuli were presented.

sponse. For the purposes of analysis, data from the first test trial with each stimulus were combined across the two test sessions for the sated and nonsated conditions. Inspection of these data reveal that the correct response was less likely in the presence of the stimuli in which it had previously earned the sated outcome ( 6.5 responses per minute) than during the stimuli in which it had produced the nonsated outcome ( 9.0 responses per minute). An analysis of these data found this difference to be statistically significant $[T(16)=26.5, p<.05]$. That correct responding was sensitive to differences in the value of the outcome predicted by the discriminative cue suggests that the rats learned about the identity of the outcome. Such a result is clearly not conducive to an $S-R$ analysis of biconditional discriminative performance.

In summary, the results of Experiment $1 \mathrm{~B}$ do not appear to favor accounts of biconditional discrimination 
learning in terms of the binary models outlined in the introduction. The fact that performance of the correct response was not selectively disrupted by modifying the information that the discriminative stimulus provided about the occurrence of its outcome suggests that response is not promoted by the $\mathrm{S}-\mathrm{O}$ association. Second, the observation that changing the value of the outcome earned by that response does affect its performance suggests that responding is not controlled through an $S-R$ association. These results were confirmed and strengthened by Experiments 2 and 3 .

\section{EXPERIMENT 2}

The purpose of Experiment 2 was to examine further the impact of outcome devaluation on biconditional discrimination performance. The design of the biconditional task used in Experiment 1B was modified in order to increase its sensitivity to the effect of outcome devaluation. In Experiment 1B, the two biconditional tasks employed different outcomes but used the same pair of instrumental responses. Thus, each response was associated with both outcomes. Colwill and Rescorla (1990a) have reported that a discriminative stimulus will encourage performance of its response even after the outcome with which they share an association has been devalued, provided there is some valued consequence associated with that response. Thus, in Experiment 1B, the continued attractiveness of one outcome may have provided support for performance of the response in the presence of the stimulus in which it had previously earned the currently devalued (i.e., sated) outcome. To eliminate this source of support for responding, the subjects were again trained on two separate biconditional discriminations, but each employed a different pair of instrumental responses.

The basic design of Experiment 2 is shown in Figure 5. Following acquisition of the two biconditional discriminations, the value of one of the outcomes was reduced by pairing the outcome with a nausea-inducing agent. The subjects were then tested in extinction on each biconditional discrimination. The question of interest is whether performance of the correct response will be depressed when the outcome used to train that response has been devalued. Such a result is clearly not anticipated if biconditional stimuli control their responses through S-R connections.

\section{Method}

\section{Subjects and Apparatus}

Sixteen experimentally naive Holtzman-derived Sprague-Dawley rats served as subjects. They were housed and maintained under the same conditions as the subjects in Experiment 1A. The apparatus used was the same as that employed in Experiment $1 \mathrm{~A}$.

\section{Procedure}

Magazine training. The subjects received one session of magazine training in which 10 food pellets followed by 10 liquidsucrose outcomes were delivered on a VT $60-\mathrm{sec}$ schedule. No response manipulanda were available.

Response training. All subjects were trained to lever press, chain pull, nose poke, and handle pull for either food-pellet or liquid-sucrose rewards. The responses were trained in separate sessions on a CRF schedule until 30 reinforcers had been earned. The order of training was lever press, chain pull, nose poke, and handle pull.

Following CRF training, each response was trained on a VI 30 -sec schedule for two 20 -min sessions. The order of training was handle pull, nose poke, chain pull, and lever press for the first four sessions. This sequence was reversed for the next four sessions. For half of the animals, pellets served as the reinforcer for lever press and chain pull responses, and sucrose served as the reinforcer for nose poking and handle pulling. These responseoutcome combinations were switched for the remaining animals.

Biconditional discrimination training. The subjects were trained on two biconditional discrimination tasks, one using the visual cues ( $\mathrm{L}$ and $\mathrm{F}$ ) and one using the auditory cues ( $T$ and $\mathrm{N}$ ). Within each task, each stimulus uniquely signaled which one of two concurrently available responses would be reinforced and which one would not. For half of the animals, lever press and chain pull were trained with the visual cues, and nose poke and handle pull with the auditory cues; the remaining animals had the opposite combination of response and stimulus pairs. For all animals, one response from each pair was designated correct in one stimu-

\section{Basic Design of Experiment 2}

Training

A1: R1-O1, R2A2: R2-O1, R1-

V1: R3-O2, R4 V2: R4-O2, R3-
Devaluation

\begin{tabular}{|c|}
\hline $\mathrm{O} 1+, \mathrm{O} 2-$ \\
\hline $\mathrm{O} 2+, \mathrm{O} 1-$ \\
\hline
\end{tabular}

Testing

\author{
A1: R1 v R2 \\ A2: R1 v R2 \\ V1: $R 3$ v R4 \\ V2: R3 v R4
}

Figure 5. Basic design of Experiment 2. A1 and $\mathrm{A2}$ denote auditory discriminative cues (noise and tone), and V1 and V2 denote visual discriminative cues (steady light and flashing light). R1, R2, R3, and R4 are instrumental responses (nose poke, handle pull, lever press, and chain pull). $\mathrm{O} 1$ and $\mathrm{O} 2$ are reinforcers (food pellets and liquid sucrose). + and - denote whether the outcome was devalued or not. 
lus and the other response was correct during the other stimulus. Correct responses were reinforced on a VI $30-\mathrm{sec}$ schedule with the same outcome used for their initial training; incorrect responses and ITI responses were never reinforced. Thus, pellets served as the reinforcer for one biconditional discrimination and sucrose served as the reinforcer for the other biconditional discrimination.

The specific stimulus, response, and outcome combinations that were distributed across subjects were as follows. Four animals were given pellets for chain pulling in $\mathrm{N}$ and lever pressing in $\mathrm{T}$ and were given sucrose for nose poking in $\mathrm{L}$ and handle pulling in F. Four other animals were given pellets for nose poking in $\mathrm{N}$ and handle pulling in $\mathrm{T}$ and were given sucrose for chain pulling in $\mathrm{L}$ and lever pressing in $\mathrm{F}$. Another four animals received sucrose for lever pressing in $\mathrm{N}$ and chain pulling in $\mathrm{T}$ and received pellets for handle pulling in $\mathrm{L}$ and nose poking in $\mathrm{F}$. The remaining four animals received sucrose for handle pulling during $\mathrm{N}$ and nose poking during $T$ and received pellets for lever pressing in $\mathrm{L}$ and chain pulling in $\mathrm{F}$.

There were 12 sessions of training on the task with the visual biconditional stimuli and 12 sessions on the task with the auditory biconditional stimuli. Each session contained 1630 -sec presentations of each biconditional stimulus. For each task, the mean duration of the ITI was $15 \mathrm{sec}$ for the first session, $30 \mathrm{sec}$ for the next 5 sessions, $60 \mathrm{sec}$ for the following 3 sessions, and finally $90 \mathrm{sec}$ for the final 3 sessions.

Outcome devaluation. There were five 2 -day cycles of outcome devaluation training. On the first day of each cycle, the tobe-devalued outcome was delivered on a VT 60 -sec schedule for $20 \mathrm{~min}$ or until five reinforcers had accumulated in the food magazine. The subjects were then injected with a $5 \mathrm{ml} / \mathrm{kg}$ solution of $0.6 \mathrm{M}$ lithium chloride $(\mathrm{LiCl})$ administered intraperitoneally before being returned to their home cages. On the second day of each cycle, the other outcome was delivered on a VT 60-sec schedule. At the end of this session, no injection was administered and the subjects were simply returned to their home cages. The doors of the sound-attenuating boxes were propped open for the last two conditioning cycles in order to permit observation of the animals' consumption of the outcomes. During this period, the room lights were dimmed.

Testing. Each pair of biconditional discriminative stimuli was tested with its original response pair. In each test session, there were eight $30-\mathrm{sec}$ presentations of each of the two stimuli with a $30-\mathrm{sec}$ ITI. For all subjects, the lever and chain were available in the first test session, and the nose poke and handle pull were available in the second test session. During testing, responding was never reinforced.

Testing was repeated following training designed to elevate overall levels of responding. That training involved one $20-\mathrm{min}$ session with each of the four responses reinforced on a VI $30-\mathrm{sec}$ schedule with the valued outcome. This was followed by one 20-min session with each of the two pairs of responses in which each response was reinforced with the valued outcome on a VI $60-\sec$ schedule. The test procedure was identical to that described above, except that the order of the two tests was reversed.

\section{Results and Discussion}

\section{Biconditional Discrimination Training}

Both biconditional discriminations were acquired successfully in that a response occurred most often during the stimulus in which it was reinforced. Analysis of performance in the final session of discrimination training collapsed across stimulus, response, and outcome identity revealed that performance of the correct response (16.9 responses per minute) was significantly higher than performance of the incorrect response $(4.2$ responses per minute) during the biconditional stimuli $[T(16)=0, p<.01]$. Furthermore, relative to the ITI rate (3.0 responses per minute), the biconditional cues significantly elevated performance of both correct responses $[T(16)=0, p<.01]$ and incorrect responses $[T(16)=20, p=.01]$.

The assignment of which outcome was to be devalued was balanced with respect to the various stimulus, response, and outcome combinations. In addition, terminal biconditional discrimination performance was matched so that there were no significant differences in responding as a function of whether or not the outcome was to be devalued. The mean correct, incorrect, and ITI response rates, respectively, were $16.1,4.0$, and 3.1 responses per minute for the to-be-devalued outcome and $17.6,4.4$, and 2.9 responses per minute for the nondevalued outcome.

\section{Outcome Devaluation}

Outcome devaluation was accomplished smoothly. Over the course of the conditioning cycles, the subjects began to reject the outcome that was paired with the toxin but continued to consume the nondevalued outcome. By the end of this phase, no animal consumed the devalued sucrose and all but three animals showed complete rejection of the devalued pellets. All subjects consumed the valued outcome. In the consumption test administered after the second test session, all animals rejected the devalued outcome.

\section{Testing}

The results of primary interest from the first extinction tests with the discriminative stimuli and their original responses are shown in Figure 6. Responding trained with the devalued outcome is shown on the left of Figure 6 , and responding trained with the valued outcome

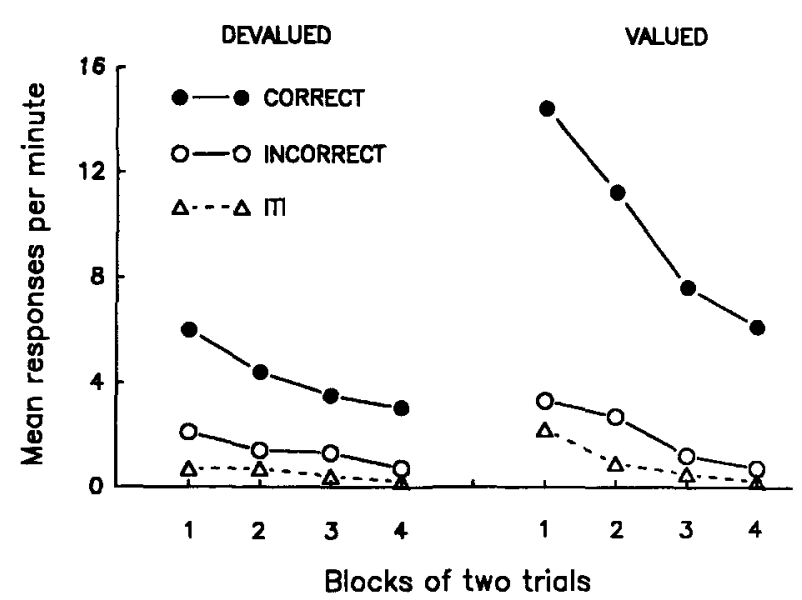

Figure 6. Experiment 2: Mean rates of responding on the biconditional discriminations in the first extinction test after the training outcome had been devalued (left panel) or not (right panel). In each panel, performance is shown separately for correct responses (filled circles) and incorrect responses (open circles) during the discriminative stimuli and during the ITI (open triangles) when no stimuli were delivered. 


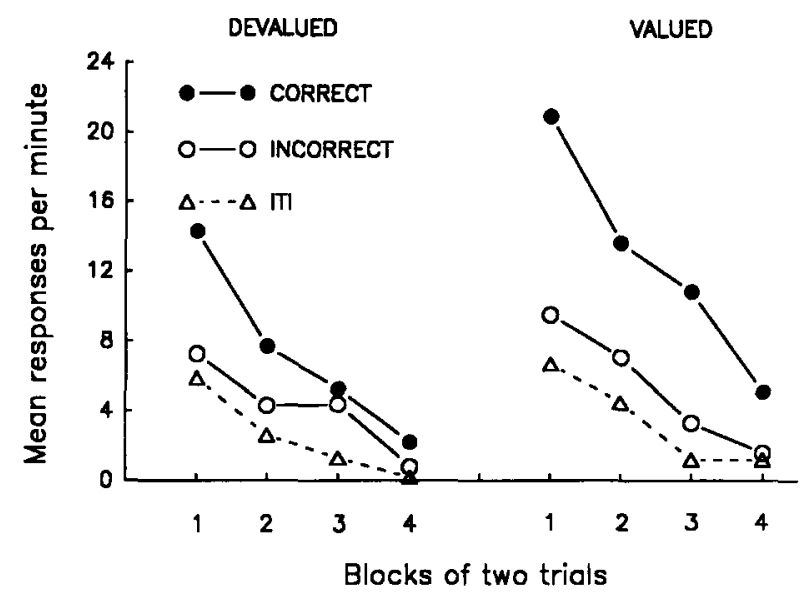

Figure 7. Experiment 2: Mean rates of responding on the biconditional discriminations in the second extinction test after the training outcome had been devalued (left panel) or not (right panel). Prior to this test, all responses had been given VI training with the valued outcome. In each panel, performance is shown separately for correct responses (filled circles) and incorrect responses (open circles) during the discriminative stimuli and during the ITI (open triangles) when no stimuli were delivened.

is shown on the right of Figure 6. In each case, performance is shown separately for the correct response, the incorrect response, and during the ITI. It is clear that devaluation of the outcome produced a substantial decrease in performance of the correct response trained with that outcome. The mean rate of correct responses was significantly lower for the devalued outcome than for the nondevalued outcome $[T(16)=19.5, p<.01]$. Neither comparison of incorrect responses nor comparison of ITI responses revealed any significant effect of the outcome value.

Biconditional discrimination performance continued to show sensitivity to the value of the current outcome even after performance of the devalued response had been elevated by training with the valued outcome. The results of that test are shown in Figure 7 . There were no significant differences in either overall ITI rates or overall incorrect response rates as a function of the value of the outcome. However, in the test session, the mean rate of correct responses that had earned the outcome whose value had been reduced was significantly lower than the rate of correct responses trained with the currently valued outcome $[T(16)=21.5, p<.05]$. These results confirm those of Experiment $1 \mathrm{~B}$ and show that responses trained in a biconditional discrimination are affected by changes in the value of their instrumental outcomes. Such sensitivity of instrumental performance to the current value of the outcome is clearly not consonant with a classical S-R analysis of biconditional discrimination learning.

\section{EXPERIMENT 3}

On the basis of the results of the present experiments, it is clear that rats have knowledge about the identity of the reward used in a biconditional discrimination and that they use this knowledge to guide performance of the correct response. Furthermore, the finding in Experiment $1 \mathrm{~B}$ that subsequent training of the biconditional cue as an S-did not selectively undermine performance of the correct response renders implausible an explanation for performance of that response in terms of the product of $\mathrm{R}-\mathrm{O}$ and $\mathrm{S}-\mathrm{O}$ binary associations. The purpose of Experiment 3 was to assess the generality of this finding by combining the design of Experiment 2 with the S- extinction manipulation of Experiment 1B.

The basic design of Experiment 3 is outlined in Figure 8 . The subjects were trained concurrently on two independent biconditional discriminations. As in Experiment 2, each discrimination employed a different pair of responses and a unique rewarding outcome. Following acquisition of both discriminations, the four biconditional cues were established as signals for the nonreinforcement of a different response, displacement of a joystick. In the absence of the stimuli, joystick responses earned food pellets for half of the animals and sucrose liquid for the remaining animals. Thus, the biconditional cues for one task signaled the omission of an outcome that was the same as that used in original biconditional discrimination training, whereas the cues for the other task signaled the omission of a different outcome. Each pair of biconditional cues was then tested with its original pair of responses. It was anticipated that if the biconditional discriminations had been solved using binary $\mathrm{S}-\mathrm{O}$ and $\mathrm{R}-\mathrm{O}$ associations then $\mathrm{S}-$ training would produce an outcome-dependent disruption of correct responses in those discriminations.

\section{Method}

Subjects and Apparatus

Sixteen experimentally naive Holtzman-derived SpragueDawley rats served as subjects. They were housed and maintained under the same conditions as subjects in Experiment $1 \mathrm{~A}$. The apparatus used was the same as that employed in Experiment 1 A with one additional response, a joystick manipulandum (Model 80111, Lafayette Instrument). This manipulandum consisted of a steel pole, measuring approximately $12.1 \mathrm{~cm}$ in length and $0.6 \mathrm{~cm}$ in diameter, inserted through a hole in the ceiling of the operant chamber. The end of the pole was approximately $6 \mathrm{~cm}$ above the grid floor and $7 \mathrm{~cm}$ from the center of the end wall opposite the food magazine. A response was recorded whenever the joystick was displaced from its centered position. Access to the joystick was prevented by removing the joystick from the operant chamber.

\section{Procedure}

Magazine and response training. The procedures for magazine training and initial training of lever pressing, chain pulling, nose poking, and handle pulling were identical to those described for Experiment 2.

Biconditional discrimination training. As in Experiment 2, the subjects were trained on two biconditional discrimination tasks, one using the visual cues and one using the auditory cues. Within each task, each stimulus uniquely signaled which one of two concurrently available responses would be reinforced and which one would not. Details about the counterbalancing of various combinations of stimuli, responses and outcomes across animals were identical to those of Experiment 2 . In addition, the same 
procedure used for training the subjects in Experiment 2 was used to establish discriminative performance in this experiment.

$\mathbf{S}-$ training. In this phase, the four biconditional discriminative stimuli were trained as signals for the nonreinforcement of a joystick response that otherwise earned pellets for half of the animals and sucrose for the other animals. To this end, all subjects were initially trained to displace a joystick for either food pellets or sucrose liquid. In the first session, each joystick response earned a reward. Responding was then reinforced on a VI 30 -sec schedule for five 20-min sessions.

Following this training, the subjects received two types of $\mathrm{S}-$ training sessions distributed randomly across days. These sessions differed only in whether the auditory or the visual stimuli were presented. In each session, there were 1630 -sec presentations of each of the two biconditional cues affiliated with the same task. The order of trial presentations within a session was randomized in blocks of eight trials, and the mean ITI was $30 \mathrm{sec}$. Joystick responding was reinforced with the outcome used for its initial training on a VI 30 -sec schedule, except during stimulus presentations when no outcomes were available. The particular combinations of cue and outcome identity were counterbalanced across animals. The subjects were given 10 sessions of $S-$ training with the visual cues and 10 sessions with the auditory cues. The design of this phase ensured that for each subject the two cues for one biconditional task signaled the omission of the same outcome used for their original training and that the two cues for the other task signaled the omission of a different outcome. That arrangement makes the design maximally sensitive to any decremental effect that $\mathrm{S}$ - training with the same outcome might have by taking advantage of the fact that generalization between stimuli in the same sensory modality is likely to be greater than that between stimuli from different modalities.

Testing for control of original responses. Each pair of biconditional discriminative stimuli was tested with its original pair of responses. In each test session, there were eight 30 -sec presentations of each of the two stimuli with an ITI of $30 \mathrm{sec}$. The trial sequence followed an ABBABAAB schedule. For all subjects, the lever and chain were available in the first test session, and the nose poke and handle pull were available in the second test session. During testing, responding was never reinforced.

\section{Results and Discussion}

\section{Biconditional Discrimination Training}

Acquisition of both biconditional discriminations proceeded smoothly, with the result that responding oc- curred most often during the stimulus in which it was reinforced. Analysis of performance in the final session of discrimination training collapsed across stimulus, response, and outcome identity revealed that performance of the correct response ( 16.7 responses per minute) was significantly higher than performance of the incorrect response ( 5.3 responses per minute) during the biconditional stimuli $[T(16)=0, p<.01]$. Furthermore, relative to the ITI rate ( 2.6 responses per minute), the biconditional cues significantly elevated performance of both the correct response $[T(16)=0, p<.01]$ and the incorrect response $[T(16)=7, p<.01]$.

The selection of pellets or sucrose as the reinforcer for joystick responding was balanced with respect to the various stimulus, response, and outcome combinations. In addition, terminal biconditional discrimination performance was matched so that there were no significant differences in responding as a function of whether or not the outcome used for S- training was the same as or different from that earned in the presence of the cues. The mean correct, incorrect, and ITI response rates, respectively, were $17.4,6.0$, and 3.1 responses per minute for the same-outcome condition and $16.1,4.6$, and 2.1 responses per minute for the different-outcome condition. There were no significant differences in the rates of either correct, incorrect, or ITI responses between the same- and different-outcome conditions.

\section{S- Training}

Over the course of S- training, the level of responding during the ITI increased, whereas responding during the stimuli declined gradually. During the first session of $\mathrm{S}-$ training, the mean rates of responding during the same- and different-stimulus conditions and the ITI were $5.1,5.3$, and 4.5 responses per minute, respectively. None of these differences was significant. Analysis of the terminal S- session revealed that responding during the ITI ( 8.3 responses per minute) was significantly higher than responding during either the same stimulus (4.0 responses per minute) or the different stimulus ( 3.2

Basic Design of Experiment 3

Training

A1: R1-O1, R2-
A2: R2-01, R1-
V1: R3-O2, R4
V2: R4-O2, R3-

S- Training

Testing

\begin{tabular}{l} 
R5-O1, A1: R5-, A2: R5- \\
R5-O1, V1: R5-, V2: R5- \\
\hline R5-O2, A1: R5-, A2: R5- \\
R5-O2, V1: R5-, V2: R5-
\end{tabular}

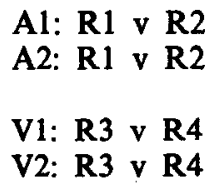

Figure 8. Basic design of S - training and the test of its effects on original biconditional diseriminative performance in Experiment 3. A1 and $\mathrm{A} 2$ denote auditory discriminative cues (noise and tone), and V1 and V2 denote visual discriminative cues (steady light and flashing light). R1, R2, R3, R4, and R5 are instrumental responses (nose poke, handle pull, lever press, chain pull, and joystick). $O 1$ and $\mathrm{O} 2$ are reinforcers (food pellets and liquid sucrose). 
responses per minute) $[T \mathrm{~s}(16)=2, p s \leq .01]$. Although the mean rate of responding during the same stimulus was slightly higher than that during the different stimulus, this difference was not significant $[T(16)=50.5$, $p>.10]$.

Suppression ratios for the first and last sessions of Straining were calculated for each subject by dividing the rate of responding during the stimulus by the sum of that rate and the ITI rate. S - training resulted in a significant increase in the suppression of responding during stimulus presentations relative to the ITI between the first and last training sessions (.52 and .30, respectively) $[T(16)=0, p<.01]$.

\section{Testing for Control of Original Responses}

Of most interest are the results of the extinction test with the discriminative stimuli and their original responses. These data are summarized in Figure 9. Responding is shown in the presence of the biconditional stimuli trained as signals for the omission of either the same outcome (top panel) or a different outcome (lower panel). Within each panel, responding is shown separately for the correct response, the incorrect response, and during the ITI. Inspection of Figure 9 reveals that the discriminative stimuli continued to exhibit differential control over their original responses regardless of which outcome had been used for $S-$ training. In the presence of stimuli trained as $\mathbf{S}-\mathbf{s}$ with the same outcome, performance of the correct response was significantly elevated relative to both the incorrect response $[T(16)=11, p<.01]$ and the ITI $[T(16)=0, p<.01]$. Similar results were obtained when the stimuli had been trained as $\mathrm{S}-\mathrm{s}$ with a different outcome. Performance of the correct response was significantly elevated relative to the rate of incorrect responses $[T(16)=2, p<.01]$ and to responding during the ITI $[T(16)=0, p<.01]$. For both the same- and the different-outcome conditions, the rates of incorrect responses were greater than their respective ITI rates $[T \mathrm{~s}(16)=5, p \mathrm{~s}<.01]$.

That biconditional control was not differentially affected by $S$ - training as a function of whether the same outcome or a different outcome was used is further supported by the results of direct comparisons of correct and incorrect response rates across the different- and same-outcome conditions. There was no significant difference between performance of the correct response $[T(16)=63.5, p>.05]$ or between the incorrect response rates $[T(16)=41, p>.10]$.

In summary, these results strengthen and extend the related findings of Experiment 1B. First, they replicate the finding that $\mathrm{S}$ - training with the same outcome has no adverse effect on the ability of biconditional stimuli to promote performance of their responses. Second, they show that the failure of $\mathrm{S}-$ training in Experiment $\mathrm{lB}$ to disrupt discriminative control was not due to masking by the association between the correct response and its other valued outcome. Thus, in contrast to a simple $S+$, a biconditional discriminative stimulus retains the ability to promote performance of the response whose rein- forcement it signals after training as a cue that another response will not be followed by that rewarding outcome.

\section{GENERAL DISCUSSION}

The results of the present experiments reveal three characteristics of biconditional discrimination learning. First, a biconditional discriminative stimulus provides information about the identity of the outcome earned in its presence. Using the transfer procedure, Experiment $1 \mathrm{~A}$ demonstrated that a biconditional cue would promote performance of a new response trained with the same outcome but not one trained with a different outcome. Second, biconditional performance does not depend upon the integrity of the information that the stimulus provides about the identity of the earned outcome. In Experiments 1B and 3, there was no specific decremental effect on performance of the original response of training a biconditional stimulus as a signal that a different response would not be followed by that outcome. Third, biconditional performance is sensitive to postlearning manipulations of the value of the outcome. Correct responding was reduced in the presence of its discriminative stimulus when the outcome was devalued using either motivational (Experiment 1B) or conditioning (Experiment 2) operations.

These results have important implications for analyses of biconditional discrimination learning in terms of binary associations. With respect to classical S-R theory, the present observations of response sensitivity to outcome devaluation confirm the inadequacy of an account of instrumental performance purely in terms of an $\mathrm{S}-\mathrm{R}$ association. However, it should be noted that other aspects of the results may appear to be consistent with the view that $\mathrm{S}-\mathrm{R}$ associations may make some contribution to performance. For instance, both the ineffectiveness of S - training in eliminating discriminative control and the residual performance following outcome devaluation are to be expected if an association exists between the stimulus and the correct response. Consequently, it is of some interest to examine the extent to which elaborations of classical S-R theory can predict the complete pattern of results obtained in the present studies.

Two-process theories were developed in an attempt to preserve the classical $S-R$ account of instrumental performance and to accommodate evidence that learning about the outcome occurs during instrumental training. The latter goal was accomplished by allowing a Pavlovian association to develop between the stimulus and the outcome. The function of this Pavlovian S-O association was to provide either additional stimulus support (Trapold \& Overmier, 1972) or motivational support (Rescorla \& Solomon, 1967) for the instrumental response. Thus, Trapold and Overmier (1972) proposed that the Pavlovian S-O association yields an outcome expectancy that becomes part of the stimulus complex associated with the response. Rescorla and Solomon (1967), on the other hand, argued that the Pavlovian $\mathrm{S}-\mathrm{O}$ asso- 

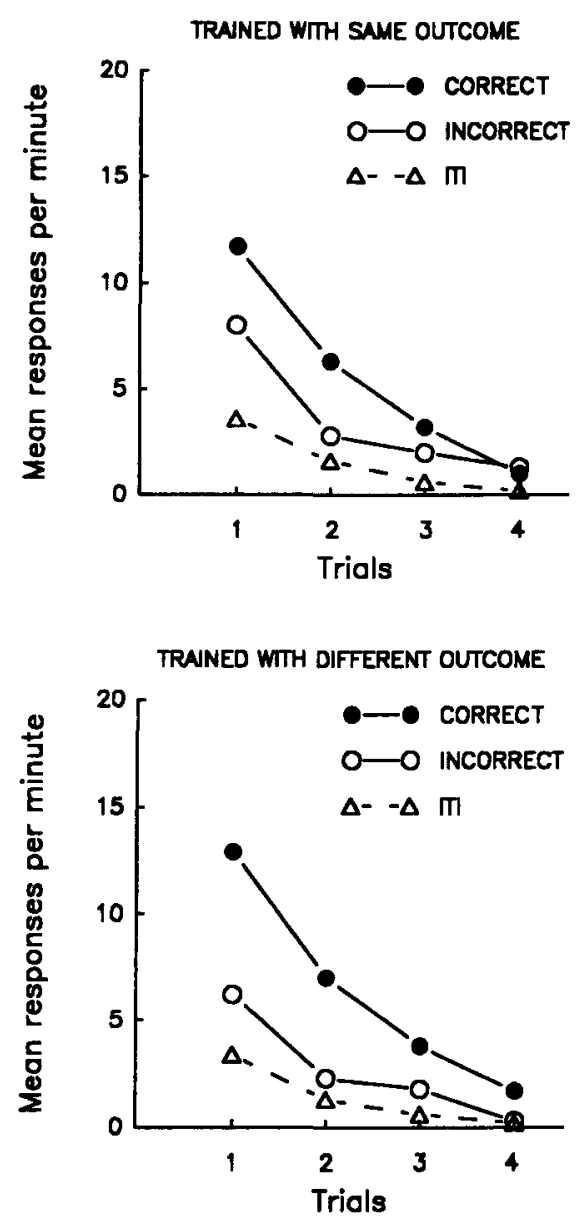

Figure 9. Mean rates of responding on the biconditional discriminations following $S$ - training in Experiment 3. The top panel displays performance on the discrimination whose cues were trained with the same outcome used for $S$ - training; the bottom panel displays the comparable data when $S$ - training employed a different outcome. In each panel, correct (filled circles) and incorrect responses (open circles) are plotted separately. Responding is also shown during the ITI (open triangles) when no stimuli were presented.

ciation simply energizes the expression of instrumental behavior. Colwill and Rescorla (1986) have commented on the ability of these two-process accounts to handle the basic demonstrations that responses are sensitive to the value of the outcomes used to train them. However, both accounts have difficulty explaining the pattern of outcome-devaluation effects obtained in the present experiments.

The predictions of the two-process theory developed by Trapold and Overmier (1972) regarding the effects of outcome devaluation in Experiments $1 \mathrm{~B}$ and 2 turn out to be indistinguishable from those made by classical S-R theory. In those experiments, each biconditional discrimination task employed only one outcome. This feature of the designs guarantees that the two discriminative stimuli within a task would each develop an association with the same outcome. Thus, according to Trapold and Overmier (1972), both cues within a task would come to evoke the same outcome expectancy. Consequently, relative to the physical features of the biconditional cues, that outcome expectancy would be uninformative about which of the two responses was to be followed by the outcome. Elsewhere, it has been reported that such incidental or redundant stimuli do not gain control over behavior (Mackintosh, 1984; Wagner, Logan, Haberlandt, \& Price, 1968). Thus, in the tasks used in the present study, the contribution of the outcome expectancy generated by the Pavlovian $\mathrm{S}-\mathrm{O}$ association to performance would be negligible. Therefore, there should have been no differential effect on instrumental responding of outcome devaluation in either Experiment IB or Experiment 2.

The pattern of devaluation effects obtained in the present experiments also poses a problem for the version of two-process theory proposed by Rescorla and Solomon (1967). According to their account, performance of both the correct and the incorrect responses should have been depressed following devaluation of the instrumental outcome. Consistent with that account is the finding that performance of the correct response was reduced in the presence of its discriminative stimulus following a decrease in the value of its outcome. However, there was no comparable effect for incorrect responding. Even after the general level of responding had been increased, the likelihood of an incorrect response in the presence of the stimulus associated with the devalued outcome was not significantly different from the likelihood of incorrect responding in the stimulus that signaled the valued outcome. Such results provide little encouragement for the idea that the observed devaluation effects were mediated by a reduction in the motivation for responding normally provided by the Pavlovian $\mathrm{S}-\mathrm{O}$ association.

Various shortcomings of two-process theories of instrumental learning have been noted on previous occasions by proponents of the view that both responses and discriminative stimuli become associated with their instrumental outcomes (Colwill \& Rescorla, 1988, 1990b; Rescorla \& Colwill, 1989). The combined R-O and S-O account of instrumental learning rightly anticipates the sensitivity that correct responses showed to manipulations of the value of their outcomes in Experiments 1B and 2. However, the finding that biconditional performance was resistant to the destructive consequences that $\mathrm{S}$ - training has been found to exert on simple discriminative control was not anticipated by this view. A graceful reconciliation of these different effects of S - training on discriminative control does not appear possible within this particular binary framework. At best, it might be argued that the conflict in signaling whether or not the same outcome will occur that is inherent in the biconditional tasks used here prevents the development of an inhibitory S-O association during subsequent $\mathrm{S}-$ training. Although there is little theoretical support for this speculation, it is relatively easy to test empirically by using different outcomes to reinforce correct responses within a biconditional task. Such training should render vulnerable to the effects of subsequent $S-$ train- 
ing whichever biconditional cue had previously signaled the outcome that was used for $\mathrm{S}-$ training. Colwill (1994) tested this prediction and found no evidence that such training of biconditional cues reduced their immunity to the disruptive effects of $S-$ training with the same outcome.

Before abandoning a binary analysis of biconditional discrimination learning, it may be useful to consider a model of instrumental learning in which the contribution to performance of the various binary connections that have been described is determined by features of the training situation. It seems quite reasonable that simple $\mathrm{S}+$ training encourages the development of predominantly $\mathrm{S}-\mathrm{O}$ and $\mathrm{R}-\mathrm{O}$ associations. However, in biconditional training, the concurrent signaling of the occurrence of $O$ for one response and its omission for another response may selectively undermine development of an $\mathrm{S}-\mathrm{O}$ association. Under these circumstances, the subject may come to rely on an $\mathrm{S}-\mathrm{R}$ association to retrieve a representation of the correct response. Performance of that response would then be determined by evaluation of the consequences of that response activated by the $R-O$ association. This combination of $\mathrm{S}-\mathrm{R}$ and $\mathrm{R}-\mathrm{O}$ associations would predict the devaluation data of Experiment 2 . Such a view would also anticipate the preservation of discriminative control following $S$ - training in Experiments $1 \mathrm{~B}$ and 3 . Unfortunately, this view has difficulty explaining the remaining results. First, a selective effect of outcome devaluation should not have been found in Experiment 1B. Although the S-R association would lead to retrieval of the correct response for that stimulus, the $\mathrm{R}-\mathrm{O}$ association would result in activation of both the valued and the devalued outcomes associated with that correct response. Consequently, there should not have been differential performance of that response. For the same reason, this view would not have predicted the transfer effect found in Experiment 1A. Because both of the outcomes associated with the response would have been activated as a consequence of the S-R and $\mathrm{R}-\mathrm{O}$ connections, there should have been no opportunity for selective control over the transfer responses. An additional point worth noting about this argument is that Colwill (1994) has found no evidence that transfer can in fact be mediated by the combination of S-R and $\mathrm{R}-\mathrm{O}$ associations. An explanation of instrumental biconditional discrimination learning in terms of binary connections does not seem very promising.

Several authors have cautioned against analyses of instrumental learning in terms of binary associations between various pairs of elements in an instrumental task (e.g., Colwill \& Rescorla, 1986; Mackintosh \& Dickinson, 1979; Skinner, 1938). They have argued instead for a single associative structure incorporating all three terms of the task. One particularly attractive version of this point of view is that a discriminative stimulus becomes associated with the particular $\mathrm{R}-\mathrm{O}$ relations arranged in its presence, $S \rightarrow(R-O)$ and $S \dashv(R-O)$. Support for this hierarchical view of instrumental learning has come from studies employing the classic switching design in which the identity of the discriminative stimulus disambiguates which responses lead to which outcomes. Specifically, two responses are reinforced with different outcomes in the presence of two different stimuli. Each stimulus, however, signals unique responseoutcome combinations. Thus, one stimulus ( $\mathrm{S} 1$ ) signals that one response (R1) leads to one outcome (O1) and that the other response (R2) leads to a different outcome $(\mathrm{O} 2)$; but the other stimulus (S2) signals the opposite combinations of responses and outcomes (i.e., R1 leads to $\mathrm{O} 2$ and $\mathrm{R} 2$ leads to $\mathrm{O} 1$ ). Because each outcome follows both responses and occurs in the presence of both stimuli, the binary associations are uninformative about which outcome follows a particular response in a given stimulus.

Recent work has documented that in this type of discrimination task rats learn about the higher order relations (Colwill \& Rescorla, 1990b; Rescorla, 1990). For example, Colwill and Rescorla (1990b) trained rats on a switching task and then made one of the outcomes unattractive. In a subsequent extinction test with the stimuli and responses, the subjects showed a preference within each stimulus for the response that had previously earned the currently attractive outcome in that stimulus. The observation that the prevailing response preference was determined by the identity of the discriminative stimulus strongly suggests that the rats had encoded the hierarchical relations.

The hierarchical model is able to provide a satisfactory explanation for the three major findings obtained in the present experiments. First, the hierarchical model attributes transfer effects to differential generalization across $\mathrm{R}-\mathrm{O}$ relations. Novel combinations of a discriminative stimulus and an $\mathrm{R}-\mathrm{O}$ relation are treated as more similar to the original training condition when both responses share an association with the same outcome. Second, the immunity of biconditional performance to the potential decremental effects of establishing the discriminative cues as $\mathrm{S}-\mathrm{s}$ for other responses is attributed to changes in the generalization gradients produced by original training. In the present experiments, each biconditional cue was simultaneously established as a signal for the reinforcement of one response and the nonreinforcement of a different response. This discrimination training may have sharpened the generalization gradients such that additional training of the cue as a signal for the nonreinforcement of yet another response would have little impact on the ability of that cue to elicit its original R-O relation (see Mackintosh, 1974). Third, the hierarchical model predicts the pattern of outcome devaluation effects found in Experiments $1 \mathrm{~B}$ and 2. Because the response-outcome relation is associated with a specific stimulus, performance of the correct response would be susceptible to the influence of a shift in the value of the outcome. The incompleteness of the devaluation effect is not necessarily in conflict with a hierarchical account of instrumental learning. Colwill and Rescorla (1990a) have offered several suggestions for the residual behavior observed following outcome de- 
valuation that are quite compatible with the general thesis that responses are associated with their outcomes.

It would be somewhat remiss not to acknowledge quite a different conceptualization of the three-term contingency that continues to be invoked to account for discrimination learning (e.g., Preston, Dickinson, \& Mackintosh, 1986; Wilson \& Pearce, 1989). This alternative assumes the learning of associations between configurations of particular stimulus and response combinations and their consequences, $\mathrm{SR} \rightarrow \mathrm{O}$ and $\mathrm{SR} \dashv \mathrm{O}$. Colwill and Rescorla (1990b) have discussed application of the configural cue account to the results of outcome devaluation on discriminative control in a switching design. The present findings that outcome devaluation affects biconditional performance pose no problem for this view. Furthermore, the failure of the outcome to mediate a selective impairment of $\mathbf{S}-$ training on biconditional control is also consistent with the predictions of the configural account. Any generalization of extinction from the SR configuration in $\mathrm{S}$ - training to the SR configurations in the biconditional discriminations should be equivalent regardless of which outcome is used in S- training. However, Colwill's (1993b) original observation that Straining with the same outcome selectively disrupts the ability of an $\mathrm{S}+$ to control its response is not predicted by the configural cue model. Even more damaging for this position are data showing that discriminative control established in a switching design may be selectively affected by manipulations of its $\mathrm{R}-\mathrm{O}$ relation in another stimulus context (Rescorla, 1990). In both cases, the configural cue account does not anticipate differential generalization between stimulus-response configurations as a function of outcome identity. It does not seem very plausible that subjects would employ only a configural cue solution to the biconditional discriminations used in the present experiments.

In this discussion, it has been assumed that a biconditional cue employs a symmetrical structure for encoding information about the consequences of both its reinforced (correct) response and its nonreinforced (incorrect) response. However, evidence for hierarchical learning has come exclusively from situations in which stimuli signal that responses will be followed by rewarding outcomes. Thus, whereas empirical confirmation exists for learning of $S \rightarrow(R-O)$ relations, there is no comparable support for $\mathrm{S} \dashv(\mathrm{R}-\mathrm{O})$ associations. In fact, analyses of signals for the nonreinforcement of instrumental responses seem to suggest that such stimuli develop direct inhibitory connections with those responses (Bonardi, 1989; Colwill, 1991). Given the present interpretation of the various effects of $S$ - training on previously established discriminative control, it is crucial that future studies inspect further the mechanisms by which biconditional cues control their incorrect responses.

In summary, the present analysis has favored the opinion that biconditional discriminations using a single outcome are solved with hierarchical associations. This conclusion is of special interest because various binary solutions to the problem were also feasible. Thus, the present data make clear that hierarchical solutions are not deployed only under conditions in which binary solutions fail to capture the accuracy of the instrumental contingencies that are in effect. Rather, it seems that at the very least hierarchical learning occurs when discriminative cues disambiguate the multiple consequences associated with an instrumental response. Whether hierarchical associations mediate all types of instrumental discriminations or whether multiple codes are employed depending upon the nature of the instrumental task is an issue that remains to be settled.

\section{REFERENCES}

BonaRDI, C. (1989). Inhibitory discriminative control is specific to both the response and the reinforcer. Quarterly Journal of Experimental Psychology, 41B, 225-242.

ColwILl, R. M. (1991). Negative discriminative stimuli provide information about the identity of omitted response-contingent outcomes. Animal Learning \& Behavior, 19, 326-336.

Colwill, R. M. (1993a). An associative analysis of instrumental learning. Current Directions in Psychological Science, 2, 111-116.

ColwILl, R. M. (1993b). Signaling the omission of a response-contingent outcome reduces discriminative control. Animal Learning \& Behavior, 21, 337-345.

Colwill, R. M. (1994). Associative representations of instrumental contingencies. In D. L. Medin (Ed.), The psychology of learning and motivation (Vol. 31, pp. 1-72). New York: Academic Press.

COLWILl, R. M., \& REscorla, R. A. (1986). Associative structures in instrumental learning. In G. H. Bower (Ed.), The psychology of learning and motivation (Vol. 20, pp. 55-104). New York: Academic Press.

COLWILl, R. M., \& Rescorla, R. A. (1988). Associations between the discriminative stimulus and the reinforcer in instrumental learning. Journal of Experimental Psychology: Animal Behavior Processes, $14,155-164$.

ColWILl, R. M., \& Rescorla, R. A. (1990a). Effect of reinforcer devaluation on discriminative control of instrumental behavior. Journal of Experimental Psychology Animal Behavior Processes, 16, 40-47.

Colwill, R. M., \& Rescorla, R. A. (1990b). Evidence for the hierarchical structure of instrumental learning. Animal Learning \& Behavior, 18, 71-82.

Hull, C. L. (1943). Principles of behavior. New York: AppletonCentury-Crofts.

MaCkINTOSH, N. J. (1974). The psychology of animal learning. London: Academic Press.

MaCKINTOSH, N. J. (1984). Conditioning and associative learning. Oxford: Oxford University Press.

Mackintosh, N. J., \& Dickinson, A. (1979). Instrumental (Type II) conditioning. In A. Dickinson \& R. A. Boakes (Eds.), Mechanisms of learning and motivation (pp. 143-167). Hillsdale, NJ: Erlbaum.

Preston, G. C., Dickinson, A., \& Mackintosh, N. J. (1986). Contextual conditional discriminations. Quarterly Journal of Experimental Psychology, 38B, 217-237.

ResCORLA, R. A. (1990). Evidence for an association between the discriminative stimulus and the response-outcome association in instrumental learning. Journal of Experimental Psychology: Animal Behavior Processes, 16, 326-334.

ResCoRla, R. A., \& Colwill, R. M. (1989). Associations with anticipated and obtained outcomes in instrumental learning. Animal Learning \& Behavior, 17, 291-303.

Rescorla, R. A., \& Solomon, R. L. (1967). Two-process learning theory: Relationships between Pavlovian conditioning and instrumental learning. Psychological Review, 74, 151-182. 
Richeson, J. A., \& ColwiLl, R. M. (1994, April). Mechanisms of response suppression by negative discriminative stimuli. Poster presented at the 65th Annual Meeting of the Eastern Psychological Association in Providence, RI.

SKINNER, B. F. (1938). The behavior of organisms. New York: AppletonCentury-Crofts.

TraPold, M. A. (1970). Are expectancies based upon different positive reinforcing events discriminably different? Learning \& Motivation, 1, 129-140.

Trapold, M. A., \& Overmier, J. B. (1972). The second learning process in instrumental learning. In A. A. Black \& W. F. Prokasy
(Eds.), Classical conditioning II. Current research and theory (pp. 427-452). New York: Appleton.

Wagner, A. R., Logan, F. A., Haberlandt, K., \& Price, T. (1968). Stimulus selection in animal discrimination learning. Journal of Experimental Psychology, 76, 171-180.

Wilson, P. N., \& Pearce, J. M. (1989). A role for stimulus generalization in conditional discrimination learning. Quarterly Journal of Experimental Psychology, 41B, 243-273.

(Manuscript received May 17, 1993; revision accepted for publication June 14, 1994.) 